\title{
Software Power Measurement of ARM Processor Based Embedded System
}

\author{
V. A. Kulkarni and G. R. Udupi
}

\begin{abstract}
Energy consumption is very crucial for battery powered Embedded Systems. In recent times, embedded system functionalities are increasing by many folds. But the battery technology is not advancing in the same pace. This necessitates the need to reduce energy consumption of battery powered embedded applications. The total energy of a typical embedded system is due to energy of software and hardware components. Once we know the total energy, we can estimate battery life accurately. Conversely, various measures can be taken to increase battery life. This paper presents measurement based approach to find energy cost associated with ARM instructions.
\end{abstract}

Index Terms - Current measurement, Embedded system, Energy consumption, software power.

\section{INTRODUCTION}

Embedded systems are being used extensively in modern life ranging from agriculture, commercial, aviation, defense to medical applications. Earlier, performance and size were design parameters for embedded systems. With increasing complexities of functionalities and end user expectation of longer battery life, power has become critical parameter for design consideration. The rate at which complexities are added is much higher compared to development rate in battery technology. The end user expects more time between successive recharges and this is possible with power aware design at various levels. This reduced power not only increases battery life, but also helps in less heat dissipation resulting in better cooling methods. A formula based on the Arrhenius Law suggests that life expectancy of component decreases $50 \%$ for every $10^{\circ} \mathrm{C}$ increase in the temperature. Thus, reducing a component's operating temperature by the same amount (consuming less energy), doubles its life expectancy [1]. Thus by reducing the software energy, not only the time between successive recharges of battery is increased, but also battery life is increased. In addition to above, lesser power consumption reduces heat dissipation, resulting in low cost packaging, cooling methods and increases device reliability.

Power consumption model of the software can be of two types: low level models and high level models. Low level models calculate power from electrical descriptions consisting circuit level, gate level, RT level. High level models deal with instruction and functional units from software point of view. High level power estimation models can be further categorized as Instruction Level Power Analysis and Functional Level Power Analysis.

Published on November 10, 2016

V. A. Kulkarni is with AMGOI, India (e-mail: vak@amgoi.edu.in).

Dr. G. R. Udupi is with GIT, India. (e-mail: gru@git.edu.in).
The methods of embedded software energy estimation can be classified are: measurement based and simulation based. In latter approach, a simulation model of the target hardware is used to run the applications and calculate the consumption of energy. Non availability of simulation model of all hardware modules and their cost, if available, are the main constraints. Measurement based approach use data obtained from actual measurements. Since real values are obtained from target flat form, the results are more accurate [2]

At basic level, software energy can be calculated by finding energy associated with each instruction. Extremely small execution time and non-availability of precise meter (at affordable cost) for current measurement are the constraints. To overcome this, authors have proposed to execute instruction in infinite loop. The average current taken by the processor while executing an instruction, is indication of energy consumption of that instruction. For accurate results, instantaneous current to be measured [3], [4], [5].

We can express the average power consumed by a microprocessor while running a program as product of average current and voltage

$$
\mathrm{P}=\mathrm{I} \times \mathrm{V}
$$

Where $\mathrm{I}$ is the average current and $\mathrm{V}$ is the supply voltage. The energy consumed by an instruction can be expressed as

$$
\mathrm{E}=\mathrm{P} \times \mathrm{N} \times \mathrm{T}
$$

Where $\mathrm{N}$ is the number of clock cycles taken by the instruction and $\mathrm{T}$ is the clock period. Thus to estimate power / energy cost of the instruction, current drawn by the processor while executing the instruction need to be measured. Different methods of current measurement are discussed in [6].

\section{ENERGY CONSUMPTION IN PIPELINED PROCESSOR}

In the pipeline processor operation, instructions are pushed in the pipeline. Decoding and execution is carried out. In each clock cycle the number of different instructions which are processed, equals the number of the pipeline stages of the processor. Each pipeline stage processes a different instruction consuming a corresponding amount of energy. The sum of the energy consumed in all the pipeline stages corresponds to the energy consumed by the processor in that clock cycle. A test instruction is executed in a loop so that the current can be measured and same instruction is executed in different stages of pipeline viz. fetch, decode and execute. Assume a set of instructions with only one test instruction, and with the others being the reference instruction (NOP), running through the pipeline. Then in $n$ clock cycles one test instruction and $n-1$ reference instructions are executed [8]. 
Instruction energy cost consists of base instruction cost, inter instruction cost and other costs. Base instruction cost is the energy taken by an instruction (test instruction) after executing reference instruction. To find base instruction cost, loops including a test instruction and number of reference instructions are executed in the processor. Fig. 1 shows pipeline states in a three stage pipeline processor. As seen from the figure, the instruction is executed in three cycles. The current is monitored in each clock cycle. This current along with the number of cycles taken by each instruction gives an idea about the total energy. Certain factors also affect base instruction cost and are termed as energy sensitive factors. Register numbers, register values, immediate values and operand values are some of energy sensitive factors [8]. Energy cost due to operand values can be calculated by first calculating energy with zero operand and then with non-zero operands.

\begin{tabular}{|l|l|l|l|l|l|}
\hline $\begin{array}{l}\text { Pipe } \\
\text { Line } \\
\text { Stages }\end{array}$ & \multicolumn{5}{|c|}{ 3- Stage pipeline operation } \\
\hline IF & NOP & NOP & Instruction & NOP & NOP \\
\hline ID & NOP & NOP & NOP & Instruction & NOP \\
\hline EX & NOP & NOP & NOP & NOP & Instruction \\
\hline $\begin{array}{l}\text { Clock } \\
\text { Cycles }\end{array}$ & n-1 & n & n+1 & n+2 & n+3 \\
\hline
\end{tabular}

Fig. 1. Pipeline stages during instruction execution.

Inter instruction cost is due to switching activity which is a function of the present inputs and the previous state of the circuit. That is, sequence in which an instruction is executed also affects the total energy. The inter instruction effects cost can be measured by loops consisting of instruction pairs. To find inter instruction cost of two instructions, say I1 \& I2, the instruction pair is placed in an infinite loop. The current drawn is always larger than the average of the I1 and I2. The difference is the overhead cost of I1 and I2. The total energy consumed by a program is the sum of the base and overhead costs, over all the instructions executed [8]. For example, if ADD consumes $2 \mathrm{~nJ}$ and SUB consumes 1 $n J$, the sum of ADD and SUB is 3nJ but if we run ADD and SUB together, the energy consumed might be $3.5 \mathrm{~nJ}$. This $0.5 \mathrm{~nJ}$ difference is called inter instruction cost. The instruction sequence for measuring the inter-instruction effect is shown in Fig. 2. In the example shown, in four clock cycles one $I 1$, one $I 2$ and two reference instructions are executed [8].

\begin{tabular}{|c|c|c|c|c|c|}
\hline Pipe & \multicolumn{5}{|c|}{ 3- Stage pipeline operation } \\
\hline IF & NOP & $\begin{array}{l}\text { Instruct } \\
\text { on1 (I1) }\end{array}$ & \begin{tabular}{|l} 
Instruction \\
2 (I2)
\end{tabular} & NOP & NOP \\
\hline ID & NOP & NOP & \begin{tabular}{|l|} 
Instruct \\
ion1
\end{tabular} & $\begin{array}{l}\text { Instruct } \\
\text { ion2 }\end{array}$ & NOP \\
\hline $\mathbf{E X}$ & NOP & NOP & NOP & $\begin{array}{l}\text { Instruct } \\
\text { ion1 }\end{array}$ & $\begin{array}{l}\text { Instruct } \\
\text { ion2 }\end{array}$ \\
\hline $\begin{array}{l}\text { Clock } \\
\text { Cycles }\end{array}$ & n-1 & $\mathbf{n}$ & $n+1$ & $n+2$ & $n+3$ \\
\hline
\end{tabular}

Fig. 2. Pipeline states for measuring inter instruction cost.

Other costs are mainly due to resource constraints, which results in increased execution time. Effect of cache misses and stall cases are to be considered for accurate energy calculation. Cache miss can be taken into account by adding certain cycle penalty to the instruction execution time. Stall can be due to pipeline stalls or Prefetch buffer stalls.

Thus for any given program, $P$, its overall energy cost, is given by:

$$
\mathbf{E}_{\mathbf{P}}=\sum_{\mathbf{i}}\left(\mathbf{B}_{\mathbf{i}} \mathbf{x} \mathbf{N}_{\mathbf{i}}\right)+\sum_{\mathbf{i}, \mathbf{j}}\left(\mathbf{O}_{\mathbf{i}, \mathbf{j}} \mathbf{x} \mathbf{N}_{\mathbf{i}, \mathbf{j}}\right)+\sum_{\mathbf{k}} \mathbf{E}_{\mathbf{k}}
$$

In the above equation, the three terms represents base cost, inter instruction cost and other costs respectively. The base cost $\mathrm{Bi}$ of each instruction $i$, along with the number of times it is executed, $\mathrm{Ni}$, gives the base cost of the program. The inter instruction cost is given by the second term of above equation. (i,j) represents pair of consecutive instructions and $N i, j$,the number of times the pair is executed . The other costs like cache misses and stalls is taken care in third term of the equation [7] [8].

\section{PROPOSED METHOD}

Experiments were carried out to find the first two factors of instruction energy cost viz. base instruction cost and inter instruction cost. ARM Cortex-M4 based microcontrollers for an embedded application is used to find base instruction cost and inter instruction cost. The device includes $104 \mathrm{~KB}$ of on-chip SRAM, $512 \mathrm{~KB}$ on-chip flash, five generalpurpose timers, one State-Configurable Timer with PWM capabilities (SCTimer/PWM), one RTC/alarm timer, one 24-bit Multi-Rate Timer (MRT), a Windowed Watchdog Timer (WWDT), four USARTs, two SPIs, three Fast-modes plus $\mathrm{I}^{2} \mathrm{C}$-bus interfaces with high-speed slave mode, and one 12-bit 5 Msamples/sec ADC.

The ARM Cortex-M4 is a 32-bit core that offers system enhancements such as low power consumption, enhanced debug features, and a high level of support block integration. The ARM Cortex-M4 CPU incorporates a 3-stage pipeline, uses Harvard architecture with separate local instruction and data buses as well as a third bus for peripherals, and includes an internal Prefetch unit that supports speculative branching. The ARM Cortex-M4 supports single-cycle digital signal processing and SIMD instructions [21].

A loop with reference instruction, NOP, was executed. Current observed to be $2.411 \mathrm{~mA}$. To know the impact of one of the energy sensitive factors, i.e. immediate operand, three immediate values are taken. The values taken are ffff, 0000 and 1111. These are designated as high, low and medium respectively. The average of these three is taken as energy cost of the instruction.

Typical current waveform during the measurement is shown in fig. 3.

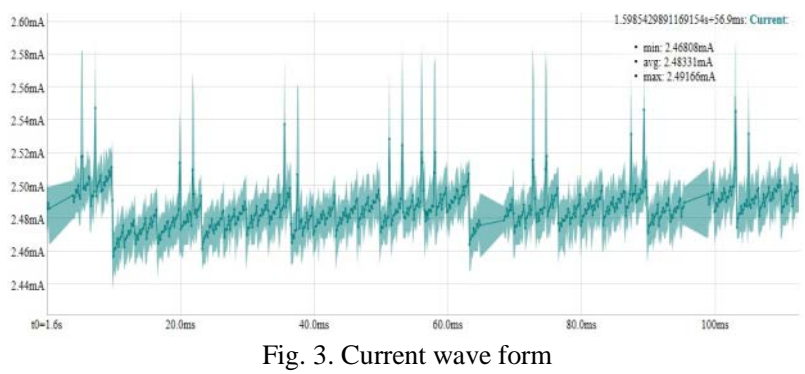


IV. RESULTS - BASE COST

TABLE I: CURRENT FOR VARIOUS INSTRUCTIONS

\begin{tabular}{|l|l|l|l|l|}
\hline $\begin{array}{l}\text { Instruct } \\
\text { ion }\end{array}$ & High (mA) & Low (mA) & $\begin{array}{l}\text { Medium } \\
(\mathrm{mA})\end{array}$ & $\begin{array}{l}\text { Average } \\
(\mathrm{mA})\end{array}$ \\
\hline ADC & 0.262708 & 0.185014 & 0.2666 & 0.238108 \\
\hline ADD & 0.271849 & 0.233308 & 0.310542 & 0.2719 \\
\hline RSB & 0.228807 & 0.252362 & 0.248781 & 0.243316 \\
\hline SBC & 0.43582 & 0.309569 & 0.33914 & 0.36151 \\
\hline SUB & 0.365007 & 0.3258373 & 0.361597 & 0.350814 \\
\hline BIC & 0.210533 & 0.173932 & 0.176555 & 0.187007 \\
\hline EOR & 0.208858 & 0.176418 & 0.125294 & 0.17019 \\
\hline AND & 0.061092 & 0.167964 & 0.285443 & 0.1715 \\
\hline ORR & 0.304444 & 0.173258 & 0.385717 & 0.287806 \\
\hline TEQ & 0.261304 & 0.261697 & 0.370976 & 0.297993 \\
\hline TST & 0.255792 & 0.460324 & 0.358854 & 0.358323 \\
\hline CMN & 0.161726 & 0.349371 & 0.286725 & 0.265941 \\
\hline CMP & 0.213936 & 0.402314 & 0.313235 & 0.309828 \\
\hline MOV & 0.401287 & 0.247479 & 0.256429 & 0.301731 \\
\hline MVN & 0.237844 & 0.395606 & 0.375503 & 0.336318 \\
\hline
\end{tabular}

The readings of Table I are taken by enabling various condition code flags. Sample readings for ADC instruction (in $\mathrm{mA}$ ) is given in Table II.

TABLE II: CURRENT FOR VARIOUS CONDITION CODES

\begin{tabular}{|c|c|c|c|c|l|}
\hline Instr & cc & high & \multicolumn{1}{c|}{ low } & medium & \multicolumn{1}{|c|}{ Average } \\
\hline ADC & cc & 0.11135 & 0.60937 & 0.16843 & 0.296383 \\
\hline ADC & cs & 0.80559 & 0.13957 & 0.17759 & 0.37425 \\
\hline ADC & eq & 0.29347 & 0.12731 & 0.56324 & 0.328007 \\
\hline ADC & ge & 0.10896 & 0.1259 & 0.15205 & 0.12897 \\
\hline ADC & hi & 0.10822 & 0.12497 & 0.14875 & 0.127313 \\
\hline ADC & le & 0.24475 & 0.13251 & 0.44005 & 0.272437 \\
\hline ADC & ls & 0.10741 & 0.12287 & 0.15245 & 0.127577 \\
\hline ADC & lt & 0.11011 & 0.12878 & 0.15278 & 0.130557 \\
\hline ADC & mi & 0.35281 & 0.30117 & 0.19348 & 0.282487 \\
\hline ADC & ne & 0.685 & 0.15264 & 0.15609 & 0.331243 \\
\hline ADC & pl & 0.11886 & 0.1276 & 0.73096 & 0.325807 \\
\hline ADC & vs & 0.10597 & 0.12748 & 0.16333 & 0.13226 \\
\hline average & 0.262708 & 0.185014 & 0.2666 & 0.238108 \\
\hline
\end{tabular}

Fig. 4-7 shows current (in $\mathrm{mA}$ ) consumed by different types of instructions for three types of immediate operands viz. high-ffff, low-0000 and medium-1111.

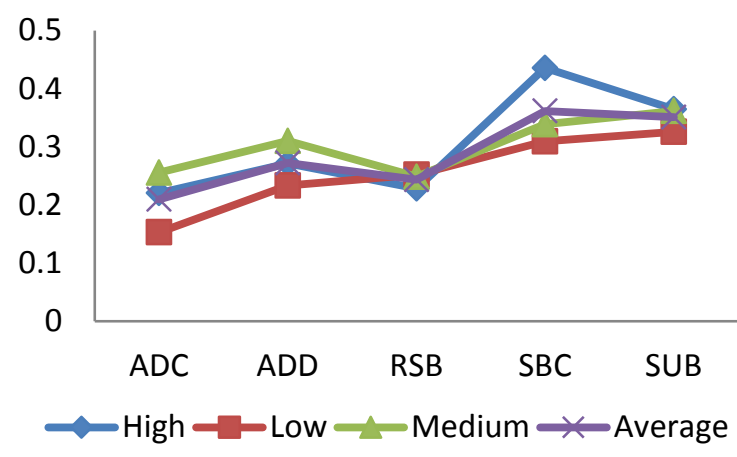

Fig. 4. Current wave form for arithmetic instructions

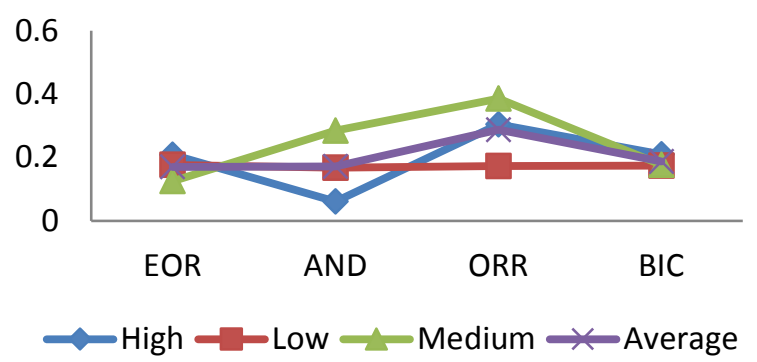

Fig. 5. Current wave form for logical instructions

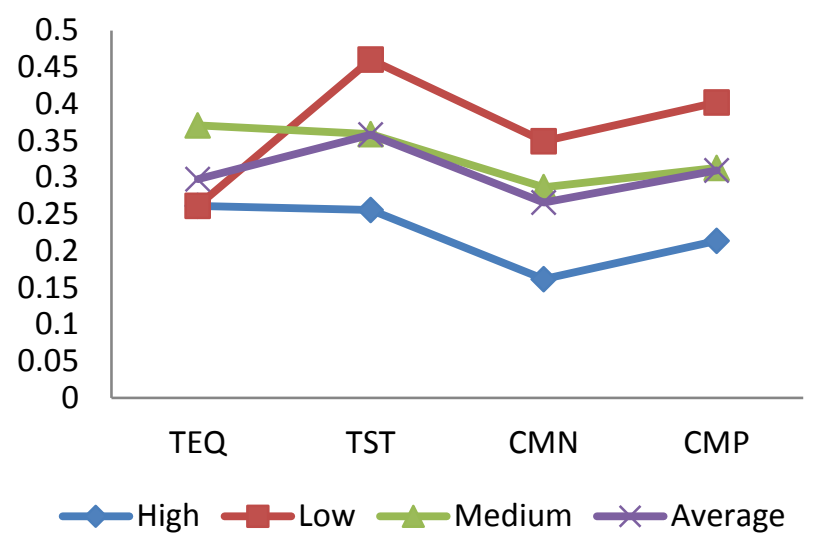

Fig. 6. Current wave form for comparison instructions

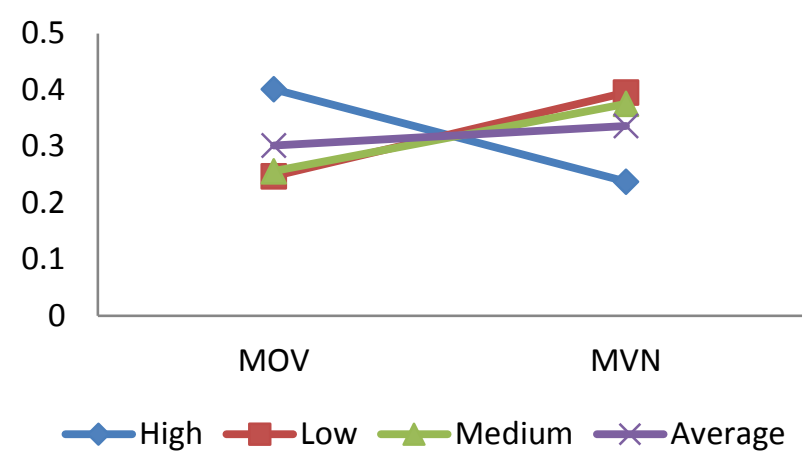

Fig. 7. Current wave form for data movement instructions

The average current (in $\mathrm{mA}$ ) taken by different types of instructions is shown in Fig.8. 


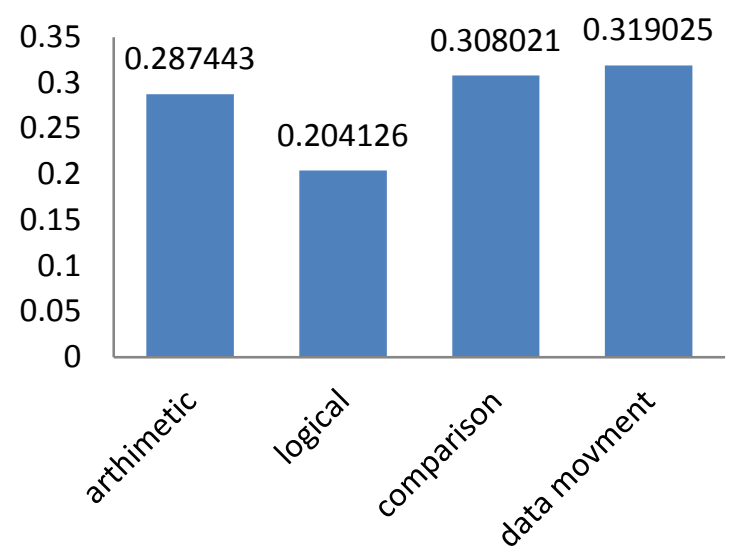

Fig. 8. Average current of different types of instructions

\section{RESULT - INTER INSTRUCTION COST}

To find inter instruction cost, measurements were carried out for different combinations of instructions. (Fig. 2) As first step, Instruction-1 was fixed and readings were taken for different data processing instructions. A sample of ADD with other instructions is shown in Table III.

TABLE III: INTER INSTRUCTION COST FOR ADD INSTRUCTION
\begin{tabular}{|c|c|c|}
\hline Instr.1 & Instr.2 & Current in mA \\
\hline ADD & ADC & 0.154 \\
\hline ADD & MOV & 0.135 \\
\hline ADD & SUB & 0.152 \\
\hline ADD & AND & 0.11 \\
\hline ADD & EOR & 0.129 \\
\hline ADD & RSB & 0.164 \\
\hline ADD & SBC & 0.174 \\
\hline ADD & RSC & 0.256 \\
\hline ADD & BIC & 0.184 \\
\hline ADD & CMP & 0.3 \\
\hline ADD & CMN & 0.233 \\
\hline ADD & TST & 0.241 \\
\hline ADD & TEQ & 0.26 \\
\hline ADD & ORR & 0.233 \\
\hline ADD & MVN & 0.347 \\
\hline
\end{tabular}

Average of inter instruction cost for ADD instruction was found to be $0.2048 \mathrm{~mA}$. Similar readings were taken for other data processing instructions i,e for different Instruction 2, current was observed for given Instruction 1. Fig. 9 shows the readings obtained.

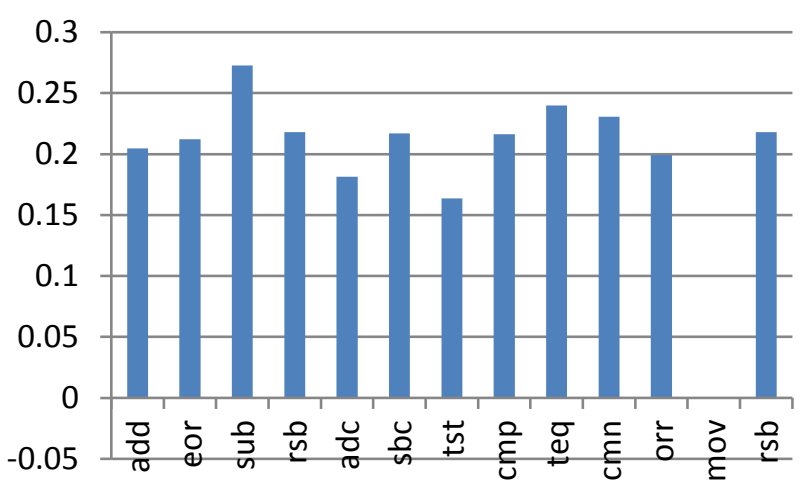

Fig. 9. Inter instruction cost

11 different addressing modes of ARM data processing instructions are shown in Table IV. TABLE IV: AdDRESSING MODES FOR ARM DATA PROCESSING
INSTRUCTIONS

\begin{tabular}{|c|c|}
\hline Mode & Addressing Mode \\
\hline 1 & ASR by Register \\
\hline 2 & ASR by Immediate \\
\hline 3 & ROR by Register \\
\hline 4 & ROR by Immediate \\
\hline 5 & LSL by Register \\
\hline 6 & LSL by Immediate \\
\hline 7 & LSR by Register \\
\hline 8 & LSR by Immediate \\
\hline 9 & Register \\
\hline 10 & Immediate \\
\hline 11 & RRX \\
\hline
\end{tabular}

Inter instruction cost between addressing modes of the same data-processing instruction is measured. The readings obtained for ADD instruction is give in Table V. Similar readings were obtained for other instruction also.

TABLE V: INTER INSTRUCTION COST BETWEEN ADDRESSING MODES OF SAME INSTRUCTION

\begin{tabular}{|c|c|c|c|c|c|}
\hline instr1/instr2 & ADD(1) & ADD(2) & ADD(9) & ADD(10) & ADD(11) \\
\hline $\mathrm{ADD}(1)$ & -0.298 & -0.0264 & -0.456 & -0.344 & 0.078 \\
\hline $\mathrm{ADD}(2)$ & 0.078 & 0.12 & -0.27 & 0.056 & -0.206 \\
\hline $\mathrm{ADD}(9)$ & -0.032 & 0.06 & -0.132 & 0.07 & 0.112 \\
\hline $\mathrm{ADD}(10)$ & 0.09 & -0.016 & 0.08 & 0.092 & 0.134 \\
\hline $\mathrm{ADD}(11)$ & 0.092 & 0.134 & 0.114 & 0.092 & 0.134 \\
\hline
\end{tabular}

\section{CONCLUSION}

Energy estimation at early stages of design will be very much useful for the designer from power budget point of view. An attempt has been made in this paper to measure base cost and inter instruction cost. Future research method would be to measure base cost and inter instruction cost for all instructions, develop a model for software energy and compare the actual values with value obtained from bench mark programs. 


\section{REFERENCES}

[1] R. Ge, et al., "Performance-constrained Distributed DVS Scheduling for Scientific Applications on Power-aware Clusters," presented at the Proceedings of the 2005 ACM/IEEE conference on Supercomputing, 2005.

[2] Mostafa Bazzaz, Mohammad Salehi and Alireza Ejlali, “An accurate instruction level energy estimation model and tool for embedded system,” IEEE trans. Instrumentation and Measurement, Vol. 62, No. 7, pp.1927-1934, July 2013.

[3] Theodore Laopoulos, Periklis Neofotistos, C. A. Kosmatopoulos and Spiridon Nikolaidis "Measurement of Current Variations for the Estimation of Software-Related Power Consumption” IEEE trans. Instrumentation and Measurement, Vol. 52, No. 4, pp.1206-1212, Aug. 2003.

[4] Nikolaos Kavvadias, Periklis Neofotistos, Spiridon Nikolaidis, C.A.Kosmatopoulos and Theodore Laopoulos, "Measurements Analysis of the Software Related Power Consumption in Microprocessors” IEEE trans. Instrumentation and Measurement, Vol. 53, No. 4, pp.1106-1112, Aug. 2004.

[5] Vasilios Konstantakos, K. Kosmatopoulos, Spiridon Nikolaidis and Theodore laopoulos, "Measurement of Power Consumption in Digital Systems” IEEE trans. Instrumentation and Measurement, Vol. 55, No. 5, pp.1662-1670, Oct, 2006.

[6] Z. Nakutis, "Embedded systems power consumption measurement methods overview”, ISSN 1392-1223, MATAVIMAL, pp 29-35, 2009.

[7] V.A.Kulkarni and G.R.Udupi, "Instruction Level Energy Estimation for Embedded System,” presented at the International Conference on Current Research and Applications in Electrical Science (ICCRAES16), PDACE, Kalburgi, India, October 04-05,2016.

[8] S.Nikolaidis, et al., " Instruction level power measurements and analysis”, Energy Aware System on Chip design of the HIPERLAN/2 standard, 2002.

[9] P. Cunha, "Energy consumption and execution time estimation of embedded system applications”, Microprocessors Microsyst., vol. 35, no. 4, p. 426 - 440, 2011. [Online]. Available: http://dx.doi.org/ 10.1016/j.micpro.2010.08.006.

[10] P. V. Joshi, K. S. Gurumurthy, "Software power analysis forembedded DSP software”, in Proc. of the Intl. Conf. on Advances in Computing and Information Technology, (ACIT 2014), Bangkok, Thailand,2014.

[11] V. Konstantakos, A. Chatzigeorgiou, S. Nikolaidis, T. Laopoulos, "Energy consumption estimation in embedded systems", IEEE Trans. Instrum. Meas., vol. 57, no. 4, p. 797-804, 2008. [Online]. Available: http://dx.doi.org/10.1109/TIM.2007.913724

[12] Sinha, A. Chandrakasan, "Software energy profiling", in Power Aware Computing, New York, Springer US, 2002, pp. 339-359. [Online]. Available: $\quad$ http://dx.doi.org/10.1007/978-1-4757-6217$\underline{4} 17$

[13] S. Nikolaidis, N. Kavvadias, P. Neofotistos, "Base instruction cost measurements", in Instruction level power measurements and analysis, Thessaloniki, Energy-Aware SYstem-on-chip design of the HIPERLAN/2 standard, 2002, pp. 14-15

[14] S. Nikolaidis, N. Kavvadias, P. Neofotistos, "Inter-instruction effect cost measurements", in Instruction level power measurements and analysis, Thessaloniki, Energy-Aware SYstem-on-chip design of the HIPERLAN/2 standard, 2002, pp. 15-16.

[15] K. Roy, M. C. Johnson, "Software design for low power", in Low Power Design in Deep Submicron Electronics, New York, Springer US, 1997, pp. 433-459. [Online]. Available: http://dx.doi.org/10. 1007/978-1-4615-5685-5_15

[16] R. Basmadjian, H. de Meer, "Evaluating and modeling power consumption of multi-core processors", in Third Int. Conf., Future Energy Systems: Where Energy, Computing and Communication Meet (e-Energy), 2012 Madrid, 2012. [Online]. Available: http://dx.doi.org/ 10.1145/2208828.2208840

[17] Mohammad Salehi and Alireza Ejlali, “A Hardware Platform for Evaluating Low-Energy Multiprocessor Embedded Systems Based on COTS Devices”, IEEE TRANSACTIONS ON INDUSTRIAL ELECTRONICS, VOL. 62, NO. 2, FEBRUARY 2015, pp 1262-1269.

[18] L. Eeckhout and K. D. Bosschere. Early design phase power/performance modeling through statistical simulation. In Intl. Symposium on Performance Analysis of Systems and Software. IEEE, 2001.

[19] W. Fornaciari, P. Gubian, D. Sciuto, and C. Silvano. Power estimation of embedded systems: A hardware/software codesign approach. In Very Large Scale Integration (VLSI) Systems, 1998.

[20] T. Givargis, F. Vahid, and J. Henkel. Instruction-based system-level power evaluation of system-on-a-chip peripheral cores. Very Large Scale Integration (VLSI) Systems, 2002.

[21] UM10850, LPC5410x User manual, available at http://www.nxp.com

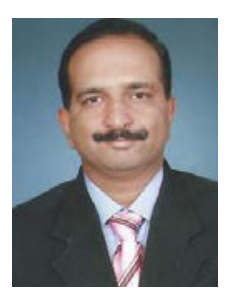

V.A.Kulkarni obtained M.Tech in VLSI design and Embedded Systems. He is pursuing Ph.D. in Visvesvaraya Technological University-Belagavi, Karnataka, India. Currently working as Associate professor and Dean (Academics) in Ashokrao Mane Group of Institutions, Maharashtra, India. He has published research articles in reputed international peer reviewed journals. He is having 20 years of teaching experience.

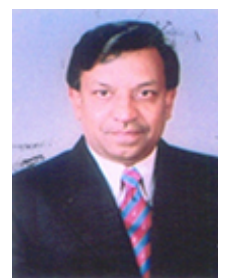

Dr. G.R.Udupi is working as Professor in Department of Electronics and Communication Engineering, Gogte Institute of Technology, Belgavi, India. He is having 33 years of teaching experience. His area of interest includes Fuzzy logic, ANN, Biomedical instrumentation, Power quality. $\mathrm{He}$ is having $20+$ international journal papers to his credit He has supervised the completion of $02 \mathrm{PhDs}$ and is guiding 06 research scholars. He is member of IEEE, BMSI, IETE and IE(I) 